\title{
Enzymatic synthesis of quercetin oleate esters using Candida antarctica lipase B
}

\begin{abstract}
Objectives: To investigate the lipase-catalyzed acylation of quercetin with oleic acid using Candida antarctica lipase B. Results: Three acylated analogues were produced: quercetin 4'-oleate $\left(\mathrm{C}_{33} \mathrm{H}_{42} \mathrm{O}_{8}\right)$, quercetin 3',4'-dioleate $\left(\mathrm{C}_{51} \mathrm{H}_{74} \mathrm{O}_{9}\right)$ and quercetin 7,3', $4^{\prime}$ trioleate $\left(\mathrm{C}_{69} \mathrm{H}_{106} \mathrm{O}_{10}\right)$. Their identities were confirmed with UPLC-ESI-MS and ${ }^{1} \mathrm{H}$ NMR analyses. The effects of temperature, duration and molar ratio of substrates on the bioconversion yields varied across conditions. The regioselectivity of the acylated quercetin analogues was affected by the molar ratio of substrates. TLC showed the acylated analogues had higher lipophilicity (152\% increase) compared to quercetin. Partition coefficient (log P) of quercetin 4'-oleate was higher than those of quercetin and oleic acid. Quercetin 4'-oleate was also stable over 28 days of storage. Conclusions: Quercetin oleate esters with enhanced lipophilicity can be produced via lipase-catalyzed reaction using $C$. antarctica lipase B to be used in topical applications.
\end{abstract}

Keyword: Acylated quercetin; Lipase; Oleic acid; Quercetin; Quercetin 4'-oleate; Regioselectivity 